\title{
Averaged Modeling of Non-ideal Boost Converter Operating in DCM
}

\author{
Guang-jun Xie, Senior Member, IACSIT, Xuan Zhao, Hai-bin Fang, and Hui-fang Xu
}

\begin{abstract}
The averaged model of non-ideal Boost converter operating in discontinuous mode (DCM) circuit was studied based on the switching elements model, the equivalent circuit method, and the energy conservation law, in which the duty ratio constraint was introduced to improve the model. The open-loop transfer function was derived to build the small signal model, by adding a voltage-controlled feedback loop, the closed-loop transfer function was also derived. The simulation results showed the correctness of the model at last.
\end{abstract}

Index Terms-Boost converter, Non-ideal, Closed-loop control

\section{INTRODUCTION}

A general power switching regulation system includes power circuit (viz. switching converter) and control circuit. Both parts interact and work together. There are many kinds of control modes in power switching regulation system, such as voltage-mode control, current-mode control, current-lag-loop-mode control, charge-mode control and so on. Voltage control circuit is simple in structure, and its anti-interference ability is strong, so it is suitable for the situations which don't require high control performance of the circuit[1-3].

There is an assignable question in switch converter modeling that is the deviation between the traditional ideal model and actual circuit[4-6]. Therefore, it is very necessary to study converter switch modeling method considering the non-ideal situation.

In this paper, take Boost converter for example, we study the average circuit model of non-ideal basic converter operating in discontinuous conduction mode (DCM), and use the duty ratio constraint to improve the average model and derive transfer function, then derive the transfer function of closed-loop control model, combining with voltage control feedback loop. Both were simulated under the MATLAB tools to verify the correctness of this model.

Manuscript received May 5, 2010. This work was supported by the Intercollegiate Key Project of Nature Science of Anhui Province.

Gang-jun Xie is with School of Electronic Science and Applied Physics, Hefei University of Technology, Hefei 230009, Anhui, China (corresponding author, e-mail: gjxie8005@hfut.edu.cn).

Xuan Zhao is with School of Electronic Science and Applied Physics, Hefei University of Technology, Hefei 230009, Anhui, China.

Hai-bin Fang is with School of Electronic Science and Applied Physics, Hefei University of Technology, Hefei 230009, Anhui, China.

Hui-fang Xu is with School of Electronic Science and Applied Physics, Hefei University of Technology, Hefei 230009, Anhui, China.

\section{LARGE SIGNAL AVERAGE MODEL OF NON-IDEAL BOOST CONVERTER IN DCM}

\section{A. Large Signal Averaged Modeling of Non-ideal Boost Converter in DCM}

When we consider the parasitic parameters of the Boost converter, we can see power switch MOSFET as that ideal switch and on resistance $R_{o n}$ are in series, see diode as the series of ideal switch, forward voltage drop $V_{F}$ and on resistance $R_{F}, R_{L} 、 R_{c}$ are respectively equivalent series resistance (ESR) of filter inductance and filter capacitor. According to the energy conservation principle, the parasitic parameters of the two switch inductance are equivalent to the inductance branch. And then use the method of average model of switching element, using current-controlled current source instead of active switching elements $\mathrm{S}$, using voltage-controlled voltage source instead of passive switching elements D. So, we can get the large signal average model, shown in Figure 1.

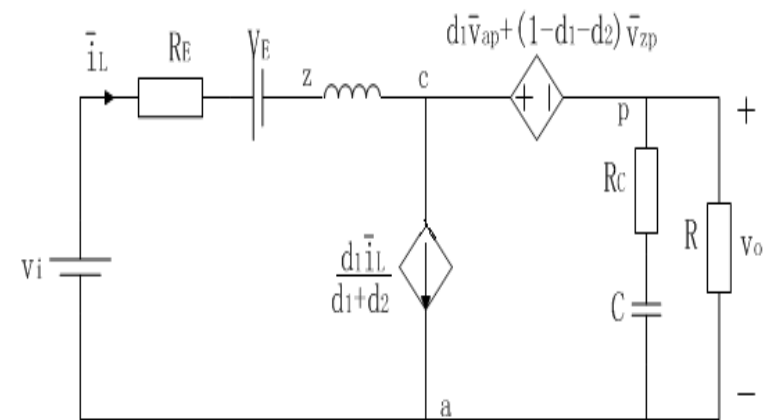

Fig.1. Large signal average model of non-ideal Boost converter in DCM

\section{B. Duty Ratio Constraint}

The duty cycle formula which is based on voltage-second of inductance voltage can not reflect accurately the frequency characteristics of the circuit, and it has limitation. This paper uses the new duty ratio constraint of Boost converter, which was derived in literature [1].

$$
d_{2}=\frac{2 L \overline{i_{L}}}{d_{1} T_{s} v_{i n}}-d_{1}
$$

The variable was analyzed to direct components and $\mathrm{AC}$ small-signal components. Then the duty ratio constraint is

$$
d_{2}=\frac{2 L \overline{i_{L}}}{d_{1} T_{s} v_{i}}-d_{1}=\frac{2 L\left(I_{L}+\hat{i}_{L}\right)}{\left(D_{1}+\hat{d}_{1}\right)\left(V_{i}+\hat{v}_{i}\right) T_{s}}-\left(D_{1}+\hat{d}_{1}\right)
$$




$$
\begin{aligned}
& \frac{d_{1} \overline{i_{L}}}{d_{1}+d_{2}}=\frac{d_{1}^{2} T_{s} v_{i}}{2 L}=\frac{\left(D_{1}+\hat{d}_{1}\right)^{2}\left(V_{i}+\hat{v}_{i}\right) T_{s}}{2 L} \\
& d_{1} \overline{v_{a p}}+\left(1-d_{1}-d_{2}\right) \overline{v_{a p}}=\left(D_{1}+\hat{d}_{1}\right)\left(V_{a p}+\hat{v}_{a p}\right) \\
& +\left[1-\frac{2 L\left(I_{L}+\hat{i}_{L}\right)}{\left(D_{1}+d_{1}\right)\left(V_{i}+\hat{v}_{i}\right) T_{s}}\right]\left(V_{z p}+\hat{v}_{z p}\right)
\end{aligned}
$$

\section{AC Small-Signal Model}

If we suppose that, the small-signal component is much less than the direct component, make the direct component is zero, and neglect the secondary small-signal product term. Then the (3) and (4) can be predigested to the (5) and (6). So we can get the small-signal linear equivalent model of non-ideal Boost converter in discontinuous conduction model, shown in fig. 2 .

$$
\begin{aligned}
& \frac{d_{1} \bar{i}_{L}}{d_{1}+d_{2}}=\frac{d_{1}^{2} T_{s} v_{i}}{2 L}=\frac{\left(D_{1}+\hat{d}_{1}\right)^{2}\left(V_{i}+\hat{v}_{i}\right) T_{s}}{2 L} \\
& =a_{1} \hat{v}_{i}+a_{2} \hat{d}_{1} \\
& d_{1} \bar{v}_{a p}+\left(1-d_{1}-d_{2}\right) \bar{v}_{z p} \\
& =-\left(D_{1}+\hat{d}_{1}\right)\left(V_{o}+\hat{v}_{o}\right)+ \\
& {\left[1-\frac{2 L\left(I_{L}+\hat{i}_{L}\right)}{\left(D_{1}+\hat{d}_{1}\right)\left(V_{i}+\hat{v}_{i}\right) T_{s}}\right]} \\
& {\left[\left(V_{i}-V_{E}-V_{o}-I_{L} R_{E}\right)+\left(\hat{v}_{i}-\hat{v}_{o}-R_{E} \hat{i}_{L}\right)\right]} \\
& =b_{1} \hat{v}_{i}+b_{2} \hat{d}_{1}+b_{3} \hat{i}_{L}+b_{4} \hat{v}_{o}
\end{aligned}
$$

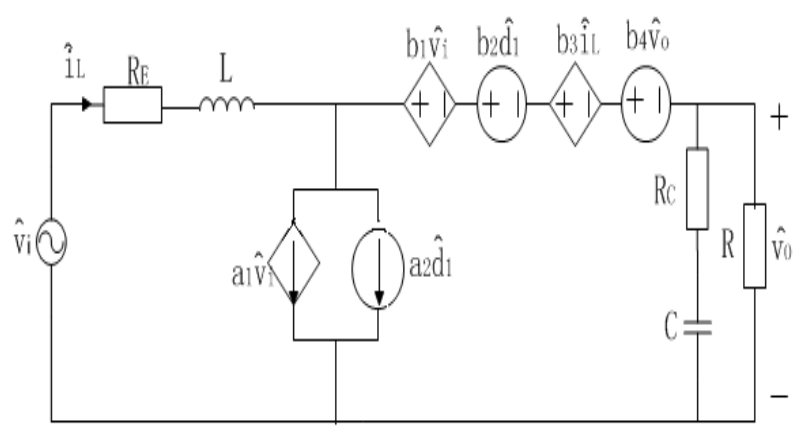

Fig.2. small-signal model of Boost converter in DCM

Form fig.2, we can calculate the input-to-output transfer function $G_{v i}(s)$ and the control-to-output transfer function $G v d(s)$ are

$$
G_{v i}(s)=\left.\frac{\hat{v}_{o}(s)}{\hat{v}_{i}(s)}\right|_{\hat{d} 1(s)=0}
$$

$$
\begin{aligned}
& =\frac{1-b_{1}-a_{1}\left(s L+R_{E}+b_{3}\right)}{1+b_{4}+\frac{\left(s L+R_{E}+b_{3}\right)\left(s R_{C} C+s R C+1\right)}{R\left(s R_{C} C+1\right)}} \\
& =\frac{t_{1} t_{4}}{t_{2} t_{3}+t_{0} t_{4}} \cdot \frac{\left(1+s / w_{z 1}\right)\left(1+s / w_{z 2}\right)}{1+\frac{s}{Q w_{0}}+\left(\frac{s}{w_{0}}\right)^{2}} \\
& =-\frac{\hat{v}_{v d}(s)=\frac{\hat{v}_{o}(s)}{\left.\hat{d}_{1}(s)\right|_{\hat{v}_{i}}(s)=0}}{1+b_{4}+\frac{\left(s L+R_{E}+b_{3}\right)\left(s R_{C} C+s R C+1\right)}{R\left(s R_{C} C+1\right)}} \\
& =\frac{t_{4} t_{5}}{t_{2} t_{3}+t_{0} t_{4}} \cdot \frac{\left(1+s / w_{z 2}\right)\left(1-s / w_{z 3}\right)}{s L} \\
& 1+\frac{s}{Q_{w_{0}}}+\left(\frac{s}{w_{0}}\right)^{2}
\end{aligned}
$$

The parameters of the actual Boost converter as following: input-voltage $V_{i}=5 \mathrm{~V}$, output-voltage $V_{o}=10 \mathrm{~V}$, Load current $\quad I_{o}=0.2 \mathrm{~A} \quad, \quad R=50 \Omega \quad, \quad L=19.2 \mu \mathrm{H}$ $R_{L}=3.6 m \Omega, C=1000 \mu F, R_{C}=2 m \Omega$. The model number of the MOSFET which we used in this paper is 2SK2690. $R_{o n}=10 m \Omega$, for Schottky diode $R_{F}=1 m \Omega$, switching frequency $f_{s}=50 \mathrm{kHz}$, PWM outputs the Peak voltage is $V_{m}=1 V$. Then there parameters are put into the formulas as following

$R_{E}=\frac{4}{3\left(D_{1}+D_{2}\right)}\left(R_{L}+\frac{D_{1} R_{o n}}{D_{1}+D_{2}}+\frac{D_{2} R_{F}}{D_{1}+D_{2}}\right)$

$V_{a p}=-V_{o}$,

$D_{2}=\frac{2 L I_{L}}{D_{1} T_{s} V_{i}}-D_{1} \quad I_{L}=\frac{I_{O}}{D_{2}}\left(D_{1}+D_{2}\right)$

$R_{E}$ is the total equivalent resistance of the three parasitic resistances which were calculated to branch of inductance. Then we can get

$$
\begin{aligned}
& D_{1} \approx 0.35, \quad R_{E} \approx 22.69 \mathrm{~m} \Omega, \quad V_{E} \approx 0.184 \mathrm{~V}, \\
& I_{L} \approx 0.43 \mathrm{~A} .
\end{aligned}
$$

Put the four parameters into formula (7) and (8), then we can get formula (9) and (10).

$$
\begin{aligned}
& G_{v i}(s)=2.112 \times \frac{\left(1+\frac{s}{500000}\right)\left(1-\frac{s}{913333}\right)}{1+\frac{s}{58.42}+\left(\frac{s}{4502}\right)^{2}} \\
& G_{v d}(s)=23.696 \times \frac{\left(1+\frac{s}{500000}\right)\left(1-\frac{s}{307433}\right)}{1+\frac{s}{58.42}+\left(\frac{s}{4502}\right)^{2}}
\end{aligned}
$$




\section{Modelling OF Boost CONVERTER With Voltage FEEDBACK-LOOP CONTROL}

Non-ideal Boost converter with voltage-mode control is shown in fig.3. Control circuit is constituted by the controller, the PWM comparator, the clock circuit and the trigger. The circuit takes a sample of the output voltage, and then the sample signal as the feedback signal is put into the input, to make the input voltage stable.

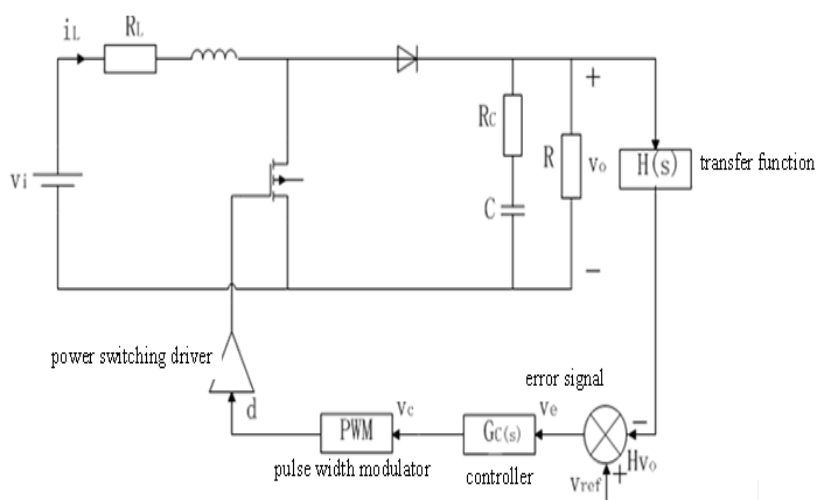

Fig.3. System structure of Boost converter with feedback-loop

\section{A. Sample Circuit and the Pulse Width Modulator}

The transfer function of the sample circuit is $H(S)$, and $H(s) v_{o} \approx v_{r e f}$, so

$$
H(s)=\frac{V_{r e f}}{V_{o}}=1
$$

The pulse width modulator is a voltage comparator actually. $v_{c}$ is the output signal of error amplifier and Compensation network.. It is compared with clock signal, then the comparator putout a pulse with the duty cycle is d, that control two main switch of the converter. The transfer function of the PWM comparator is

$$
F_{m(S)}=\frac{\hat{d}_{(S)}}{\hat{v}_{c(S)}}=\frac{1}{V_{m}}
$$

\section{B. PID Compensator}

The most important component of the design is compensator in control circuit. Transfer function of the main circuit is $G_{v d}(s)$, if there is no compensator, the transfer function $G_{c}(s)=1$, put it into formula (13), the open-loop transfer function of the system. Then we can get the open-loop transfer function (14) of the system without compensator.

$$
T(s)=\frac{H(s) G_{c}(s) G_{v d}(s)}{V_{m}}
$$

$$
\begin{aligned}
& T(s)=\frac{G_{v d}(s)}{V_{m}}= \\
& 23.7 \times \frac{\left(1+\frac{s}{500000}\right)\left(1-\frac{s}{307433}\right)}{1+\frac{s}{58.42}+\left(\frac{s}{4502}\right)^{2}}
\end{aligned}
$$

And $T(0)=23.7 \Rightarrow 27.5 \mathrm{~dB}$. We can calculate out the crossover frequency $w_{c 1}=1383 \mathrm{rad} / \mathrm{s}$ $f_{c 1}=\frac{w_{c 1}}{2 \pi}=220.2 \mathrm{~Hz}$, and the phase margin is $92^{\circ}$. It can be seen that, the crossover frequency is too low, response speed of the system is too slow, and the phase margin is too large. These are disadvantaged for else dynamic characteristics. So the compensation network is necessary. The PID compensator not only has the advantage that can improve the steady performance of system, but also has the more superiority to improve system dynamic performance. So we chose the PID compensation network, which is shown in fig. 4 .

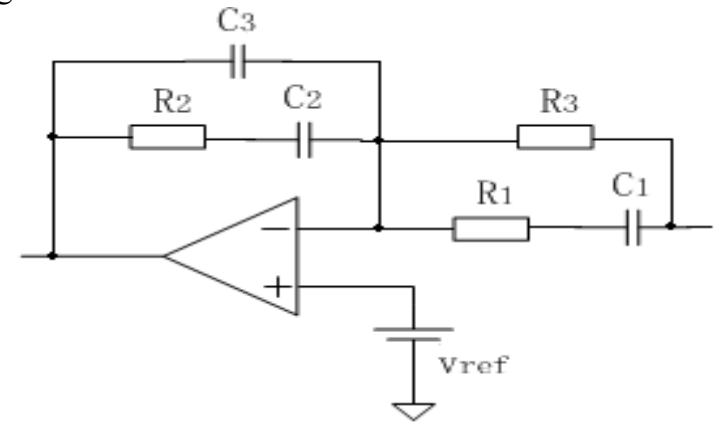

Fig.4. PID compensator in this paper

Form the fig.4, we can get the transfer function of the PID compensator, which is formula (15). Then the system Open-loop transfer function after compensated is formula (16).

$$
G_{c}(s)=G_{c 0} \frac{\left(1+\frac{s}{w_{z}}\right)\left(1+\frac{w_{L}}{s}\right)}{\left(1+\frac{s}{w_{p 1}}\right)\left(1+\frac{s}{w_{p 2}}\right)}
$$

$$
\begin{aligned}
& T_{c}(s)=G_{c}(s) T(s) \\
& =\frac{G_{c 0} G_{v d 0}}{V_{m}} \times \\
& \frac{\left(1+\frac{s}{w_{z}}\right)\left(1+\frac{w_{L}}{s}\right)\left(1+\frac{s}{w_{z 2}}\right)\left(1-\frac{s}{w_{z 3}}\right)}{\left(1+\frac{s}{w_{p 1}}\right)\left(1+\frac{s}{w_{p 2}}\right)\left[1+\frac{s}{Q w_{0}}+\left(\frac{s}{w_{0}}\right)^{2}\right]}
\end{aligned}
$$

The next step is to calculate parameters of the Compensation network. If we make $w_{p 1}=w_{z 2}=5 \times 10^{5} \mathrm{rad} / \mathrm{s}$, to cancel the zero point which was caused by output capacitance (ESR), then the 
open-loop transfer function is formula (17) now.

$$
T_{c}(s)=G_{c}(s) T(s)=\frac{G_{c 0} G_{d} d 0}{V_{m}} \frac{\left(1+\frac{s}{w_{2}}\right)\left(1+\frac{w_{L}}{s}\right)\left(1-\frac{s}{w_{23}}\right)}{\left(1+\frac{s}{w_{p 2}}\right)\left[1+\frac{s}{Q_{10}}+\left(\frac{s}{w_{0}}\right)^{2}\right]}
$$

If we make crossover frequency $f_{c}$ of open-loop transfer function after compensation, to one fifth of the switching frequency $f_{s}$, that is $f_{c}=f_{s} / 5=50 / 5=10 \mathrm{kHz}$, and suppose that phase margin is $45^{\circ}, \varphi_{m}=45^{\circ}$, then zero-point frequency and pole frequency respectively are

$$
\begin{aligned}
& f_{z}=f_{c} \sqrt{\frac{1-\sin \varphi_{m}}{1+\sin \varphi_{m}}}=10 \times \sqrt{\frac{1-\sin 45^{\circ}}{1+\sin 45^{\circ}}}=4.142 \mathrm{kHz} \\
& f_{p 2}=f_{c} \sqrt{\frac{1+\sin \varphi_{m}}{1-\sin \varphi_{m}}}=10 \times \sqrt{\frac{1+\sin 45^{\circ}}{1-\sin 45^{\circ}}}=24.142 \mathrm{kHz} \\
& \text { and make } f_{L}=\frac{f_{c}}{10}=1 \mathrm{kHz}
\end{aligned}
$$$$
\text { From }\left|T_{c}\left(j w_{c}\right)\right|_{d B}=\left|T\left(j w_{c}\right) G_{c}\left(j w_{c}\right)\right|_{d B}=0 d B \text {, we can }
$$
get

$$
\left|G_{c}\left(j w_{c}\right)\right|_{d B}=33 d B \Rightarrow 44.8
$$

Then

$$
G_{c 0}=\left|G_{c}\left(j w_{c}\right)\right| \frac{\sqrt{1+\left(f_{c} / f_{p 1}\right)^{2}} \sqrt{1+\left(f_{c} / f_{p 2}\right)^{2}}}{\sqrt{1+\left(f_{c} / f_{z}\right)^{2}} \sqrt{1+\left(f_{L} / f_{c}\right)^{2}}}
$$

$=18.6$

$$
\begin{aligned}
& G_{c}(s)=\frac{18.6\left(1+\frac{s}{26011}\right)\left(1+\frac{6280}{s}\right)}{\left(1+\frac{s}{500000}\right)\left(1+\frac{s}{151611}\right)} \\
& T_{c 0}=\frac{G_{c 0} G_{v d 0}}{V_{m}}=23.7 \times 18.6=439.1 \Rightarrow 52.8 d B \\
& T_{c}(s)=\frac{439.1\left(1+\frac{s}{26011}\right)\left(1+\frac{6280}{s}\right)\left(1-\frac{s}{307433}\right)}{\left(1+\frac{s}{151611}\right)\left[1+\frac{s}{58.42}+\left(\frac{s}{4502}\right)^{2}\right]}
\end{aligned}
$$

From

$$
w_{p 1}=\frac{1}{R_{1} C_{1}}=5 \times 10^{5} \mathrm{rad} / \mathrm{s}
$$

$$
\begin{aligned}
& w_{L}=\frac{1}{R_{2} C_{2}}=6280 \mathrm{rad} / \mathrm{s} \\
& w_{p 2}=\frac{C_{2}+C_{3}}{R_{2} C_{2} C_{3}}=151611 \mathrm{rad} / \mathrm{s}
\end{aligned}
$$

We can get $R_{1}=100 \Omega, C_{1}=20 \mathrm{nF}, \quad R_{2}=35.3 \mathrm{k} \Omega$, $C_{2}=4.5 n F, R_{3}=1.82 \mathrm{k} \Omega$, and $C_{3}=0.195 \mathrm{nF}$, respectively. And they meet the following formula.

$$
G_{c 0}=\frac{R_{2} C_{2}}{R_{3}\left(C_{2}+C_{3}\right)}=18.6
$$

\section{Simulation AND EXPERIMENTAL RESUlts}

The small-signal of open-loop and close-loop Boost converter is simulated by using MATLAB, to verify the accuracy of modeling which is proposed in this paper. The Bodes of open-loop transfer function and input-to-output transfer function are shown in fig. 5 (a), (b), respectively. Fig .6 shows the step response waveform of control-to-output in open-loop and close-loop circuit, respectively. Form the figure; we know that, crossover frequency and phase margin of open-loop transfer function in the system are more reasonable after compensation. And has decreased steady-state error in low frequency, decreased amplitude of input-to-output transfer function and output impedance in low and medium frequency, strengthened the disturbance of inhibitory ability, and improved the transient response speed and stability.

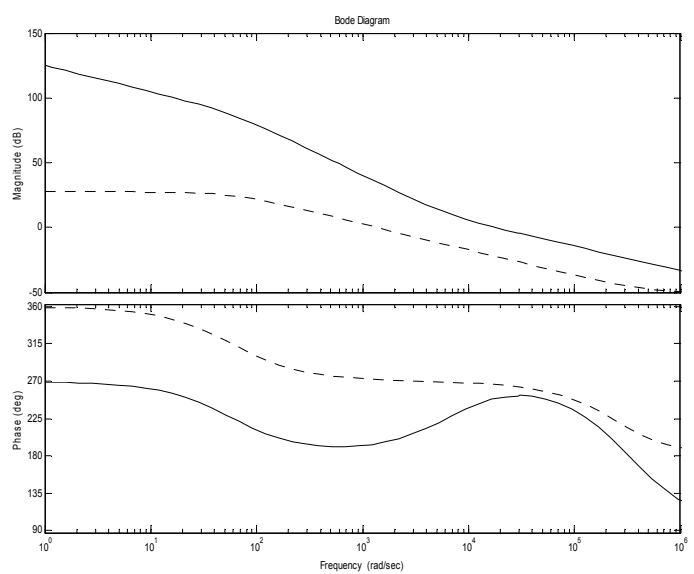

(a) The open-loop transfer function ( The broken line and real line stand for situations of before and after compensation, respectively.

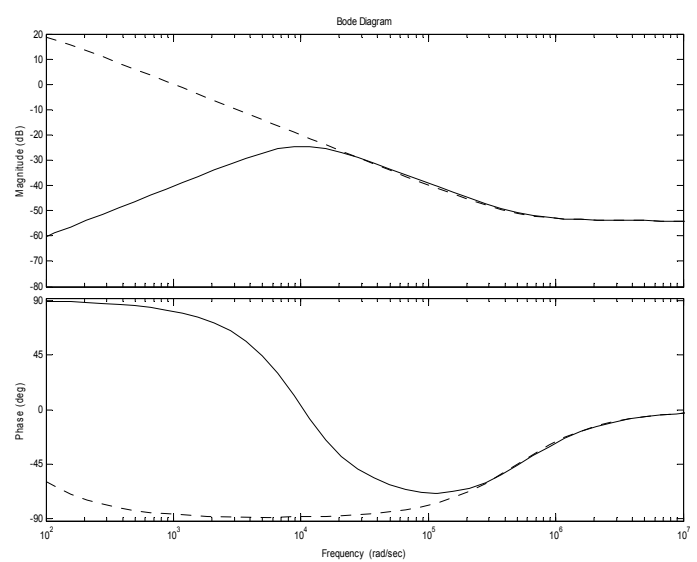

(b) The input-to-output transfer function ( The broken line and real line stand for situations of open-loop and close-loop circuits, respectively)

Fig.5. The small-signal transfer function of Boost converter 


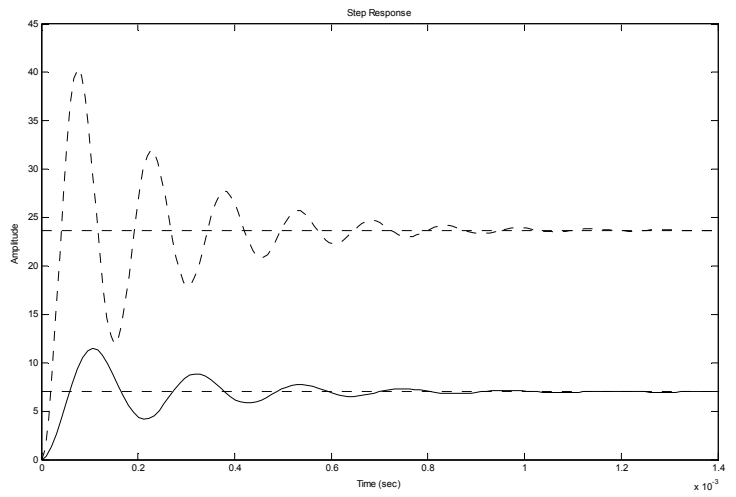

Fig.6. The step response waveform of control-to-output in open-loop and close-loop circuit. ( The broken line and real line stand for situations of before and after compensation, respectively )

\section{CONCLUSION}

Based on the average model of switching elements method, an average of the equivalent circuit method, and the idea of energy conservation law, we take Boost converter for example to study the average model of the basic non-ideal converter, which is added voltage control feedback loop in DCM circuit. And we improve the model by using the duty ratio constraint; derive the open-loop transfer function and closed-loop transfer function of the model. Both to be verified the correctness of the model building, under the MATLAB simulation tools. In this paper, we have studied the modeling of voltage-control Boost converter considered parasitic parameters in DCM. The model is useful and intuitionistic, and its physical significance is clear.

\section{REFERENCES}

[1] Sun J, Mitchell D M, Greuel M F. Averaged modeling of PWM converters operating in discontinuous conduction mode[J]. IEEE Trans. Power Electron, 2001, 16(4): 482-492.

[2] Czarkowski D, Kazimierczuk M K. Energy-conservation approach to modeling PWM dc-dc converters[J]. IEEE Trans Aerosp Electron Syst, 1993, 29(3):1059-1063.

[3] Davoudi A, Jatskevich J, Chapman P L. Averaged modelling of switched-inductor cells considering conduction losses in discontinuous mode[J]. IET Electr. Power Appl, 2007, 1(3): 402-406.

[4] Zhang Weiping. Model and Control of Switching Converter [M] Beijing: China Power Press, 2005.

[5] Wang Zhenglin, Wang Kaisheng, Chen Guozheng. MATLAB/Simulink and Control System Simulation[M]. Beijing: Electronic Industry Press, 2005.

[6] Sun J, Mitchell D M, Greuel M F. Averaged modeling of PWM converters operating in discontinuous conduction mode[J]. IEEE Trans. Power Electron, 2001, 16(4): 482-492.

Guang-jun Xie received the B.S. degree and the M.S. degree from Hefei University of Technology, in 1992 and 1995, respectively. He received the $\mathrm{Ph} . \mathrm{D}$. degree from University of Science and Technology of China in 2002. He is now a Professor of Hefei University of Technology, and his research interests include IC design, computational intelligence. 